\section{O consumo excessivo de álcool e a insatisfação com a imagem corporal por adolescentes e jovens de um município baiano, Brasil}

\author{
Excessive alcohol intake and dissatisfaction with \\ body image among adolescents and young adults \\ in a municipality in Bahia State, Brazil
}

\section{El consumo excesivo de alcohol y la insatisfacción con la imagen corporal por parte de adolescentes y jóvenes de un municipio bahiano, Brasil}

Bárbara Emanuely de Brito Guimarães 1

Rosana Aquino 2

Nília Maria de Brito Lima Prado 1

Poliana Vieira Amaral Rodrigues 3

\section{Resumo}

O estudo tem como objetivo analisar a associação entre o consumo excessivo de álcool e a insatisfação com a imagem corporal em população de adolescentes e jovens. Trata-se de um estudo transversal, tipo inquérito domiciliar, realizado com 1.582 indivíduos entre a faixa etária de 15 a 24 anos, residentes em Camaçari, Bahia, Brasil. O consumo excessivo de álcool foi identificado pelo Alcohol Use Disorder Identification Test (AUDIT), e a insatisfação com a imagem corporal, pela Escala de Figura de Silhuetas. As variáveis confundidoras foram: sexo, idade, raça, escolaridade, trabalho, religião, composição familiar, número de amigos próximos, estado conjugal e filhos. A associação entre consumo excessivo e insatisfação corporal foi estimada por meio de regressão logística. A prevalência para o consumo excessivo de álcool foi de 21,9\%, sendo maior entre o sexo masculino, e, para a insatisfação com a imagem corporal, foi de 79,5\%, sendo maior entre o sexo feminino. Não foi encontrada associação estatisticamente significante entre consumo excessivo de álcool e insatisfação com a imagem corporal. Após ajuste do modelo final, as variáveis que permaneceram associadas com o consumo excessivo foram: religião $(O R=2,02)$, escolaridade $(O R=1,63)$, composição familiar $(O R=$ $1,61)$, idade $(O R=0,55)$ e trabalho $(O R=0,61)$. Ainda que as análises não evidenciassem a associação entre o consumo excessivo de álcool e a insatisfação com a imagem corporal, os resultados deste estudo chamam a atenção para as altas prevalências em relação aos dois fenômenos. Dessa forma, evidencia-se a necessidade de desenvolvimento de intervenções voltadas às práticas de cuidado para essa população específica.

Consumo de Álcool por Menores; Adolescente; Adulto Jovem; Imagem

Corporal

\author{
Correspondência \\ B. E. B. Guimarães \\ Instituto Multidisciplinar em Saúde, Universidade \\ Federal da Bahia. \\ Rua Hormindo Barros 58, Vitória da Conquista, BA \\ 45029-094, Brasil. \\ barbaraemanuely.psi@gmail.com \\ 1 Instituto Multidisciplinar em Saúde, Universidade Federal da \\ Bahia, Vitória da Conquista, Brasil. \\ 2 Instituto de Saúde Coletiva, Universidade Federal da Bahia, \\ Salvador, Brasil. \\ 3 Prefeitura Municipal de Vitória da Conquista, Vitória da \\ Conquista, Brasil.
}




\section{Introdução}

A insatisfação com a imagem corporal é definida pela divergência de perceptiva entre o corpo real e o corpo ideal, configurando numa avaliação negativa por parte do indivíduo sobre a sua imagem corporal 1,2. A ocorrência desse fenômeno é frequente entre a população de adolescentes $(65,7 \%$ feminino e 54,5\% masculino) 3 e jovens $(64,4 \%)^{4}$. Nessas fases da vida, marcadas por transições de ordem biológica, social e psicológica 5, os indivíduos podem apresentar dificuldades em lidar e aceitar a imagem corporal 6, o que tem sido associado, na literatura científica, à preocupação em corresponder aos padrões de beleza física 3,4,7,8, sobretudo influenciada pela mídia social 9 .

Diante de altas prevalências apresentadas por estudos epidemiológicos como os realizados no interior da Bahia (80,5\%) e interior do Rio Grande do Sul (65,6\% feminino e 51\% masculino) 1,10, a insatisfação com a imagem corporal se configura como uma importante questão a ser abordada e considerada no cuidado em saúde por estar relacionada a comportamentos de risco à saúde 7,9,11,12, entre eles, o consumo excessivo de álcool 13,14,15.

O consumo excessivo de álcool tem sido discutido na literatura científica como importante preditor de comportamentos de risco à saúde, como manter relações sexuais desprotegidas, uso de substâncias ilícitas, homicídios e envolvimento em acidentes de trânsito 11,16,17,18,19 e, por conseguinte, já é considerado uma importante questão de saúde pública 20,21 .

Alguns fatores de ordem social têm sido associados ao padrão de consumo excessivo de álcool e à insatisfação com a imagem corporal, entre eles: consumir álcool com amigos 22, características individuais como: idade e sexo 12,23,24,25,26,27,28, atividade laboral 12, escolaridade 27, estado conjugal 29 e crença religiosa 30 .

Contudo, ainda são incipientes os estudos que objetivam investigar a associação entre o consumo excessivo de álcool e a insatisfação com a imagem corporal. Especialmente entre adolescentes e jovens, pesquisas sobre a temática são importantes para conhecer a dimensão do problema e colaborar com o planejamento e o desenvolvimento de ações para promoção da saúde no âmbito da atenção básica à saúde, visando potencializar a adoção de comportamentos saudáveis e reduzir os comportamentos de risco. Dessa forma, este estudo tem como objetivo analisar a associação entre o consumo excessivo de álcool e a insatisfação com a imagem corporal em população de adolescentes e jovens residentes no Município de Camaçari, Bahia.

\section{Métodos}

Com o objetivo de subsidiar as análises do presente estudo, foi elaborado um modelo teórico, tendo, como hipótese, a existência de associação entre a insatisfação com a imagem corporal (variável independente principal) e o consumo excessivo de álcool (variável dependente) entre adolescentes e jovens. As variáveis confundidoras foram classificadas em dois grupos, relacionados às características individuais: sexo, faixa etária, raça/cor, escolaridade, religião e trabalho; e às características familiares e sociais: composição familiar, número de amigos próximos, estado conjugal e ter filhos. As análises foram estratificadas pela variável sexo, apontada como importante preditor, tanto para consumo excessivo de álcool quanto para insatisfação com a imagem corporal. Ressalta-se que não é possível testar se a associação é causal, dessa forma, foi estabelecido o papel preditor para a insatisfação com a imagem corporal. Entretanto, os fenômenos estudados dificultam a possibilidade de afastar a hipótese de que, em alguma medida, o consumo excessivo, também, possa contribuir para a insatisfação corporal (Figura 1). 


\section{Figura 1}

Modelo teórico para avaliação da associação entre adolescentes e jovens em área de cobertura da atenção primária à saúde. Camaçari, Bahia, Brasil, 2011-2012.

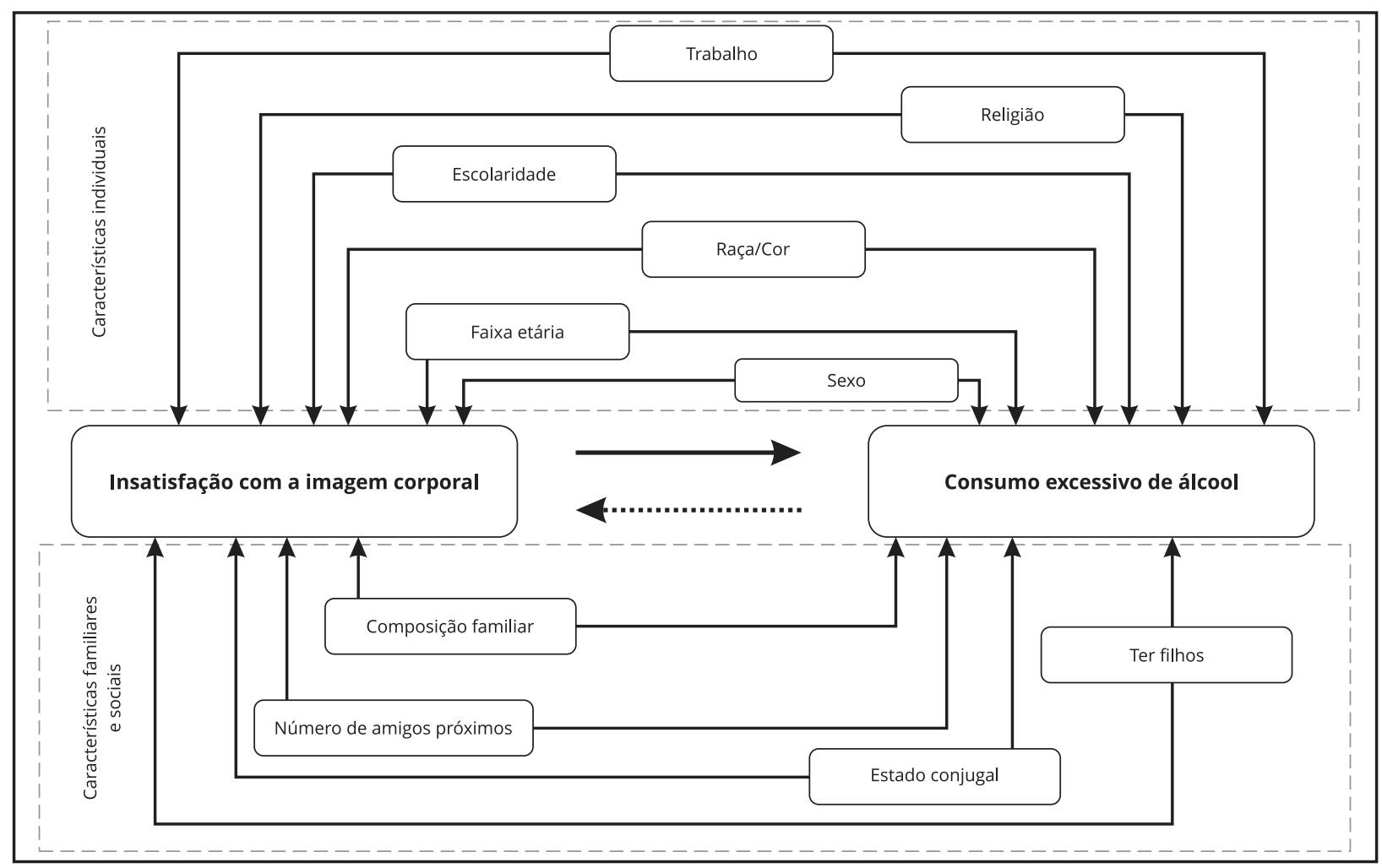

Fonte: elaborado pelas autoras.

\section{Desenho do estudo}

O presente estudo faz parte do projeto intitulado Avaliação dos Hábitos de Vida e Acessibilidade aos Serviços de Atenção Primária à Saúde da População entre 15 a 24 Anos 31. Trata-se de um estudo de corte transversal, tipo inquérito domiciliar, realizado no Município de Camaçari, no período compreendido entre outubro de 2011 a janeiro de 2012, com a população entre 15-24 anos de idade.

O Município de Camaçari fica localizado na Região Metropolitana de Salvador, ocupa uma área territorial de $784,658 \mathrm{~km}^{2}$ e tem densidade demográfica de 309,65 habitantes $/ \mathrm{km}^{2}$, sendo o quarto município mais populoso da Bahia, com população estimada, em 2010, de 242.970 habitantes 32. Em 2011, a rede de saúde era composta por 477 microáreas que representavam 100\% de cobertura do município.

As faixas etárias de 15-19 anos e de 20-24 anos correspondem, respectivamente, a 9,1\% e 10,3\% dessa população. Trata-se do município mais industrializado do estado e que possui o maior Produto Interno Bruto (PIB) industrial do Nordeste estimado em R\$ 71.012,33 (Instituto Brasileiro de Geografia e Estatística. Censo Demográfico 2010. https://censo2010.ibge.gov.br/resultados.html).

O conjunto de dados do inquérito foi obtido por meio de uma amostragem probabilística por conglomerados em dois estágios de seleção, respectivamente, microáreas (unidade primária) e indivíduos (unidade secundária). Para o primeiro estágio, foi realizado o sorteio aleatório das microáreas, com o 
intuito de obter representatividade ou diferença estatística, sendo incluídas 65 microáreas, divididas em 30 unidades de saúde da família e 35 unidades básicas de saúde.

Para a construção do segundo estágio de seleção da amostragem, inicialmente, foi realizado o sorteio dos logradouros e, em seguida, em cada logradouro sorteado, foi identificado o domicílio de menor número, sendo esse o primeiro a ser visitado para a coleta dos dados. O entrevistador seguia domicílio a domicílio em um mesmo lado da rua e, ao fim do trecho, modificava-se o trajeto para o outro lado do logradouro (se pertencesse à microárea).

Ao final, o entrevistador seguia para o próximo logradouro sorteado na microárea até completar a amostra prevista de domicílios. Após identificação dos domicílios com, pelo menos, um residente entre 15 e 24 anos, realizava-se um sorteio aleatório, quando necessário, de um morador por domicílio, nessa faixa etária, para responder ao questionário, mesmo em casos em que o indivíduo não estivesse presente no momento. Ressalta-se que gestantes, pessoas com necessidades especiais (que inviabilizassem a aplicação do questionário) e empregados domésticos, dentro da faixa etária, daqueles domicílios identificados foram excluídos do sorteio.

Informações detalhadas e precisas sobre o cálculo amostral e amostragem podem ser obtidas em Aquino et al. 31. Contudo, vale destacar que foram utilizadas, como referência para o cálculo amostral, estimativas associadas a desfecho de menor prevalência como parâmetro, e, considerado o tamanho dos conglomerados. O tamanho amostral foi estimado em 1.800 adolescentes e adultos jovens (15-24 anos), sendo essa amostra suficiente para avaliar os efeitos da Estratégia Saúde da Família na adoção de hábitos saudáveis e na acessibilidade aos serviços de atenção primária à saúde na população do Município de Camaçari em 2011 e, dessa forma, acredita-se que a abordagem deste estudo tenha poder estatístico suficiente para detectar as tais diferenças em análise. O tamanho amostral do projeto original foi composto por 1.701 indivíduos. Desses, 218 (12\%) foram excluídos do banco de dados por não possuírem informações completas referentes às variáveis de interesse desse artigo. Sendo assim, a amostra final deste estudo é composta por 1.582 indivíduos, que representam $88 \%$ da amostra total.

Foram realizadas entrevistas estruturadas e coletadas medidas antropométricas dos indivíduos em domicílio. O instrumento de coleta incluiu questões sobre características demográficas, socioeconômicas, hábitos de vida e condições de saúde.

A coleta dos dados foi precedida de estudo piloto realizado em área de abrangência de uma unidade não sorteada para o desenvolvimento da pesquisa. Todas as microáreas sorteadas foram mapeadas, utilizando-se mapas disponíveis na Prefeitura e no aplicativo Google Maps (https://www.google.com. br/maps/). Os procedimentos de coleta de dados foram padronizados pela confecção de manuais, que foram utilizados nos treinamentos da equipe e durante o trabalho de campo por supervisores e entrevistadores. A coleta foi realizada com auxílio de palmtops com sistema operacional Windows Mobile 5.0.

\section{Variáveis estudadas}

A variável dependente deste estudo, o consumo excessivo de álcool, foi identificada a partir do Alcohol Use Disorder Identification Test (AUDIT). Este teste foi desenvolvido pela Organização Mundial da Saúde, na década de 1980. No Brasil, o AUDIT foi validado e adaptado numa população geral com idade igual e superior aos 15 anos por Méndez 32 e, posteriormente, adaptado para população urbana por Lima et al. 33. O instrumento é composto por dez perguntas referentes aos 12 últimos meses, compreendidas no formato de escala Likert e, para análise, somam-se os valores referentes a cada resposta, sendo a pontuação mínima igual a 0 e a pontuação máxima igual a 40. Os indivíduos são identificados quanto ao consumo alcoólico seguindo a classificação: consumo de baixo risco ou abstinência (0 a 7 pontos); consumo de risco (8 a 15 pontos); consumo nocivo (16 a 20 pontos); possível dependência (maior ou igual a 20 pontos). O ponto de corte definido como consumo excessivo é o escore igual ou superior a 8 pontos 32,34 .

A variável independente principal foi a insatisfação com a imagem corporal, mensurada por meio da Escala de Figura de Silhuetas e definida a partir da divergência das respostas do indivíduo quanto à imagem que melhor o representa em relação à imagem que gostaria de ser 2 . No Brasil, essa escala foi criada e validada para crianças e adultos por Kakeshita et al. 35 e, posteriormente, validada para o público adolescente por Laus et al. ${ }^{36}$. É composta por 15 figuras de silhuetas variando de imagens 
mais esbeltas até imagens mais largas, para ambos os sexos 2,35,37, tendo expresso valores médios do índice de massa corporal (IMC) em cada figura 35.

As covariáveis foram agrupadas segundo as características demográficas dos indivíduos: sexo (masculino ou feminino); faixa etária (15-19 anos ou 20-24 anos); raça (branca, negra, parda e índia ou amarela); escolaridade (menor ou igual a 8 anos ou mais de 8 anos); trabalho (sim ou não) e religião (sim ou não) e características de ordem familiar e social: composição familiar (mora só, nuclear, mononuclear ou mora com outras pessoas); número de amigos próximos (nenhum ou, pelo menos, um amigo); estado conjugal (solteiro, divorciado, separado ou viúvo e casado ou mora junto) e filhos (sim ou não).

\section{Análise estatística}

Foi realizada uma análise descritiva das características da população estudada, estratificada por sexo, por meio da distribuição de frequências absolutas e relativas e utilização do teste $\chi^{2}$ de Pearson, com correção de Rao-Scott de segunda ordem para o desenho amostral, considerando a estrutura de dados de conglomerados 38,39 .

Com o objetivo de verificar a existência de associação entre o consumo excessivo de álcool e a insatisfação com a imagem corporal, foi realizada uma análise bivariada e multivariada, estratificada por sexo, utilizando modelos de regressão logística multinível, que considerou a dependência das unidades amostrais (adolescentes e jovens) dentro dos conglomerados (microáreas), calculando a odds ratio (OR) e seus respectivos intervalos de 95\% de confiança (IC95\%). As seguintes covariáveis foram utilizadas para ajuste no modelo final: faixa etária, raça, escolaridade, trabalho, religião, composição familiar, número de amigos próximos, estado conjugal e existência de filhos. A escolha das variáveis foi orientada pela construção do modelo teórico baseado na revisão da literatura científica. Para testar a robustez dos modelos, foram realizadas análises estratificadas por sexo e idade, considerando os grupos de adolescentes e jovens separadamente, mas os modelos não evidenciaram diferenças nas estimativas da associação principal investigada, sendo mantida apenas a estratificação por sexo. Todas as análises foram realizadas no software Stata versão 14.0 (https://www.stata.com). Foi considerado, em todas as análises, um nível de $5 \%$ de significância.

O projeto foi aprovado pelo Comitê de Ética em Pesquisa com Seres Humanos do Instituto de Saúde Coletiva da Universidade Federal da Bahia sob o parecer no 019/2009. Todos os entrevistados receberam esclarecimentos quanto aos propósitos do estudo e assinaram Termo de Consentimento Livre e Esclarecido. No caso dos menores de idade, além dos entrevistados, o termo também foi assinado pelos seus responsáveis, segundo as recomendações da Resolução no 196/1996 do Conselho Nacional de Saúde, vigente no período da coleta de dados. Adicionalmente, foi obtida anuência do gestor municipal de saúde do Município de Camaçari para coleta de dados.

\section{Resultados}

A amostra final deste estudo foi composta por 1.582 indivíduos. Em relação às características individuais, familiares e sociais, observa-se predominância do sexo feminino (57,4\%), com idade entre 15-19 anos (56,8\%), que autorreferiram a cor da pele como negra, parda, índia ou amarela $(89,9 \%)$, que declararam possuir religião $(63,5 \%)$, ser solteiro, divorciado ou viúvo $(78,1 \%)$, não trabalhar $(63,1 \%)$, com escolaridade maior de oito anos de estudo (70\%), não possuir filho (80,5\%), pertencer à composição familiar do tipo mononuclear, morar só ou com outras pessoas $(53,7 \%)$, ter, pelo menos, um amigo $(94,7 \%)$ e estarem insatisfeitos com a imagem corporal $(79,5 \%)$ (Tabela 1$)$.

Após a estratificação por sexo, foram observadas diferenças estatisticamente significantes entre os grupos para a maioria das variáveis estudadas, sendo maior o percentual de mulheres que declararam possuir religião (58,3\% de homens e $67,3 \%$ de mulheres), não possuir trabalho $(49,1 \%$ de homens e $73,6 \%$ de mulheres), ter mais de oito anos de escolaridade (63,1\% de homens e 75,2\% de mulheres), pertencer à composição familiar do tipo mononuclear, morar só ou com outras pessoas (47,2\% de homens e $58,5 \%$ de mulheres) e estarem insatisfeitos com a imagem corporal $(75,1 \%$ de homens e $82,8 \%$ de mulheres); enquanto foram maiores os percentuais de indivíduos do sexo masculino que 


\section{Tabela 1}

Características individuais, familiares e sociais da população de adolescentes e jovens estratificadas por sexo. Camaçari, Bahia, Brasil, 2011-2012.

\begin{tabular}{|c|c|c|c|c|c|}
\hline \multirow[t]{2}{*}{ Características } & \multicolumn{2}{|c|}{ Total } & \multirow{2}{*}{$\begin{array}{c}\text { Masculino } \\
\%\end{array}$} & \multirow{2}{*}{$\begin{array}{c}\text { Feminino } \\
\%\end{array}$} & \multirow[t]{2}{*}{ Valor de $p$ * } \\
\hline & $\mathrm{n}$ & $\%$ & & & \\
\hline Total & 1.582 & 100 & 42,6 & 57,4 & \\
\hline Idade (anos) & & & & & 0,07 \\
\hline $15-19$ & 899 & 56,8 & 59,5 & 54,8 & \\
\hline $20-24$ & 683 & 43,2 & 40,5 & 45,2 & \\
\hline Raça & & & & & 0,78 \\
\hline Branca & 159 & 10,1 & 9,8 & 10,2 & \\
\hline Negra/Parda/Índia/Amarela & 1.423 & 89,9 & 90,2 & 89,8 & \\
\hline Religião & & & & & 0,00 ** \\
\hline Não & 578 & 36,5 & 41,7 & 32,7 & \\
\hline Sim & 1.004 & 63,5 & 58,3 & 67,3 & \\
\hline Estado conjugal & & & & & 0,00 ** \\
\hline Solteiro/Divorciado/Viúvo & 1.235 & 78,1 & 90,4 & 68,9 & \\
\hline Casado/Mora junto & 347 & 21,9 & 9,6 & 31,1 & \\
\hline Trabalho & & & & & $0,00 * *$ \\
\hline Não & 999 & 63,1 & 49,1 & 73,6 & \\
\hline Sim & 583 & 36,9 & 50,9 & 26,4 & \\
\hline Escolaridade (anos) & & & & & 0,00 ** \\
\hline$\leq 8$ & 474 & 30,0 & 36,9 & 24,8 & \\
\hline$>8$ & 1.108 & 70,0 & 63,1 & 75,2 & \\
\hline Filhos & & & & & $0,00 * *$ \\
\hline Não & 1.274 & 80,5 & 92,1 & 71,9 & \\
\hline Sim & 308 & 19,5 & 7,9 & 28,1 & \\
\hline Composição familiar & & & & & $0,00 * *$ \\
\hline Nuclear & 733 & 46,3 & 52,8 & 41,5 & \\
\hline Mononuclear/Mora só ou com outras pessoas & 849 & 53,7 & 47,2 & 58,5 & \\
\hline Número de amigos & & & & & 0,02 ** \\
\hline Nenhum & 83 & 5,3 & 3,4 & 6,6 & \\
\hline Pelo menos 1 amigo & 1.499 & 94,7 & 96,6 & 93,4 & \\
\hline Insatisfação com a imagem & & & & & 0,00 ** \\
\hline Não & 324 & 20,5 & 24,9 & 17,2 & \\
\hline Sim & 1.258 & 79,5 & 75,1 & 82,8 & \\
\hline
\end{tabular}

Fonte: inquérito domiciliar, Camaçari, 2011-2012.

* Teste $\chi^{2}$ de Pearson com correção para amostragem complexa;

** Resultado estatisticamente significativo.

declararam estar solteiro, divorciado ou viúvo (90,4\% de homens e 68,9\% de mulheres), não ter filhos ( $92,1 \%$ de homens e $71,9 \%$ de mulheres) e ter, pelo menos, um amigo ( $96,6 \%$ de homens e $93,7 \%$ de mulheres) (Tabela 1).

A prevalência de consumo excessivo de álcool foi de $21,9 \%$ na amostra estudada, maior entre os indivíduos do sexo masculino $(28,6 \%)$ do que do sexo feminino (16,9\%). Tanto na amostra geral como em ambos os sexos, maiores prevalências foram observadas entre indivíduos que referiram não ter religião, estar casado ou morando junto, que tinham filhos e com composição familiar mononuclear ou que moravam só ou com outras pessoas.

Para a amostra total, também foram observadas maiores prevalências de consumo entre indivíduos de 20-24 anos, que trabalhavam e no grupo com escolaridade menor ou igual a oito anos. A associação do consumo excessivo com idade e trabalho também foi observada entre os indivíduos do 
$(\mathrm{OR}=0,62$; IC95\%: 0,47-0,82), não trabalhar ( $\mathrm{OR}=0,58$; IC95\%: 0,46-0,75) e não ter filhos ( $\mathrm{OR}=0,61$; IC95\%: 0,46-0,81) associaram-se negativamente com o consumo excessivo (Tabela 3).

Em relação à análise bivariada estratificada por sexo (Tabela 3), os fatores que apresentaram associação positiva com o consumo excessivo entre os indivíduos do sexo masculino foram: não possuir religião (OR = 1,47; IC95\%: 1,04-2,07) e composição familiar mononuclear/mora só ou com outras pessoas ( $\mathrm{OR}=2,35$; IC95\%: 1,66-3,33); enquanto que os fatores idade entre 15-19 anos ( $\mathrm{OR}=0,31$; IC95\%: 0,22-0,45), ser solteiro/divorciado/viúvo ( $\mathrm{OR}=0,27$; IC95\%: 0,16-0,46), não trabalhar $(\mathrm{OR}=$ 0,43; IC95\%: 0,30-0,62) e não ter filhos ( $\mathrm{OR}=0,35$; IC95\%: 0,19-0,62) apresentaram associação negativa com o consumo excessivo.

\section{Tabela 2}

Consumo excessivo de álcool, estratificado por sexo, segundo características sociodemográficas da população de adolescentes e jovens. Camaçari, Bahia, Brasil, 2011-2012.

\begin{tabular}{|c|c|c|c|c|c|c|}
\hline \multirow[t]{2}{*}{ Características } & \multicolumn{2}{|c|}{ Total } & \multicolumn{2}{|c|}{ Masculino } & \multicolumn{2}{|c|}{ Feminino } \\
\hline & $\%$ & Valor de p & $\%$ & Valor de p & $\%$ & Valor de $p$ * \\
\hline Total & 21,9 & & 28,6 & & 16,9 & \\
\hline Idade (anos) & & 0,00 & & $0,00 * *$ & & 0,12 \\
\hline $15-19$ & 16,9 & & 19,2 & & 15,1 & \\
\hline $20-24$ & 28,4 & & 42,5 & & 19,1 & \\
\hline Raça & & 0,30 & & 0,97 & & 0,18 \\
\hline Branca & 18,9 & & 28,8 & & 11,8 & \\
\hline Negra/Parda/Índia/Amarela & 22,2 & & 28,6 & & 17,4 & \\
\hline Religião & & 0,00 & & 0,02 ** & & $0,00 * *$ \\
\hline Não & 30,1 & & 33,1 & & 27,3 & \\
\hline Sim & 17,1 & & 25,4 & & 11,8 & \\
\hline Estado Conjugal & & 0,00 & & $0,00 * *$ & & $0,00 * *$ \\
\hline Solteiro/Divorciado/Viúvo & 20,0 & & 25,8 & & 14,4 & \\
\hline Casado/Mora junto & 28,5 & & 55,4 & & 22,3 & \\
\hline Trabalho & & 0,00 & & $0,00 * \star$ & & 0,34 \\
\hline Não & 18,4 & & 20,2 & & 17,5 & \\
\hline Sim & 27,8 & & 36,7 & & 15,0 & \\
\hline Escolaridade (anos) & & 0,00 & & 0,95 & & $0,00 * *$ \\
\hline$\leq 8$ & 27,2 & & 28,5 & & 25,8 & \\
\hline$>8$ & 19,6 & & 28,7 & & 13,9 & \\
\hline Filhos & & 0,00 & & $0,00 * \star$ & & $0,00 * \star$ \\
\hline Não & 20,1 & & 26,7 & & 13,8 & \\
\hline Sim & 29,2 & & 50,9 & & 24,7 & \\
\hline Composição familiar & & 0,00 & & $0,00 * \star$ & & $0,00 * *$ \\
\hline Nuclear & 15,8 & & 20,5 & & 11,4 & \\
\hline $\begin{array}{l}\text { Mononuclear/Mora só ou com outras } \\
\text { pessoas }\end{array}$ & 27,1 & & 37,7 & & 20,7 & \\
\hline Número de amigos & & 0,81 & & 0,75 & & 0,31 \\
\hline Nenhum & 22,9 & & 26,1 & & 21,7 & \\
\hline Pelo menos 1 amigo & 21,8 & & 28,7 & & 16,5 & \\
\hline Insatisfação com a imagem & & 0,28 & & 0,07 & & 0,2 \\
\hline Não & 24,1 & & 33,9 & & 13,5 & \\
\hline Sim & 21,3 & & 26,9 & & 17,5 & \\
\hline
\end{tabular}

Fonte: inquérito domiciliar, Camaçari, 2011-2012.

* Teste $\chi^{2}$ de Pearson com correção para amostragem complexa;

** Resultado estatisticamente significativo. 
Tabela 3

Modelo bivariado do consumo excessivo de álcool da população de adolescentes e jovens estratificado por sexo. Camaçari, Bahia, Brasil, 2011-2012.

\begin{tabular}{|c|c|c|c|c|c|c|}
\hline \multirow[t]{2}{*}{ Características } & \multicolumn{2}{|r|}{ Total } & \multicolumn{2}{|c|}{ Masculino } & \multicolumn{2}{|c|}{ Feminino } \\
\hline & OR & IC95\% & OR & IC95\% & OR & IC95\% \\
\hline \multicolumn{7}{|l|}{ Insatisfação com a imagem } \\
\hline Não & 1,00 & - & 1,00 & - & 1,00 & - \\
\hline Sim & 0,84 & $0,63-1,12$ & 0,69 & $0,47-1,02$ & 1,36 & $0,83-2,25$ \\
\hline \multicolumn{7}{|l|}{ Idade (anos) } \\
\hline $20-24$ & 1,00 & - & 1,00 & - & 1,00 & - \\
\hline $15-19$ & 0,50 & $0,39-0,64$ * & 0,31 & $0,22-0,45$ * & 0,75 & $0,53-1,06$ \\
\hline \multicolumn{7}{|l|}{ Raça } \\
\hline Branca & 1,00 & - & 1,00 & - & 1,00 & - \\
\hline Negra/Parda/Índia/Amarela & 1,22 & $0,80-1,86$ & 0,97 & $0,55-1,72$ & 1,5 & $0,82-3,02$ \\
\hline \multicolumn{7}{|l|}{ Religião } \\
\hline Sim & 1,00 & - & 1,00 & - & 1,00 & - \\
\hline Não & 2,08 & $1,63-2,66$ * & 1,47 & $1,04-2,07$ * & 2,81 & $1,97-4,00$ * \\
\hline \multicolumn{7}{|l|}{ Estado Conjugal } \\
\hline Casado/Mora junto & 1,00 & - & 1,00 & - & 1,00 & - \\
\hline Solteiro/Divorciado/Viúvo & 0,62 & $0,47-0,82$ * & 0,27 & $0,16-0,46$ * & 0,58 & $0,41-0,83$ * \\
\hline \multicolumn{7}{|l|}{ Trabalho } \\
\hline Sim & 1,00 & - & 1,00 & - & 1,00 & - \\
\hline Não & 0,58 & $0,46-0,75$ * & 0,43 & $0,30-0,62$ * & 1,20 & $0,80-1,81$ \\
\hline \multicolumn{7}{|l|}{ Escolaridade (anos) } \\
\hline$>8$ & 1,00 & - & 1,00 & - & 1,00 & - \\
\hline$\leq 8$ & 1,53 & $1,19-1,97$ * & 1,00 & $0,70-1,43$ & 2,15 & $1,49-3,10$ * \\
\hline \multicolumn{7}{|l|}{ Filhos } \\
\hline Sim & 1,00 & - & 1,00 & - & 1,00 & - \\
\hline Não & 0,61 & $0,46-0,81$ * & 0,35 & $0,19-0,62$ * & 0,49 & $0,34-0,70$ * \\
\hline \multicolumn{7}{|l|}{ Composição familiar } \\
\hline Nuclear & 1,00 & - & 1,00 & - & 1,00 & - \\
\hline Mononuclear/Mora só ou com outras pessoas & 1,97 & $1,54-2,54$ * & 2,35 & $1,66-3,33$ * & 2,03 & $1,39-2,97$ * \\
\hline \multicolumn{7}{|l|}{ Número de amigos } \\
\hline Nenhum & 1,00 & - & 1,00 & - & 1,00 & - \\
\hline Pelo menos 1 amigo & 1,07 & $0,63-1,81$ & 0,84 & $0,32-2,24$ & 1,4 & $0,74-2,66$ \\
\hline
\end{tabular}

IC95\%: intervalo de 95\% de confiança; OR: odds ratio.

Fonte: inquérito domiciliar, Camaçari, 2011-2012.

* Resultado estatisticamente significativo.

sexo masculino e, entre os indivíduos do sexo feminino, com escolaridade menor ou igual a oito anos. Não foram observadas diferenças estatisticamente significantes na prevalência de consumo quanto a raça, número de amigos e insatisfação com a imagem corporal (Tabela 2).

Nas análises bivariada (Tabela 3) e multivariada (Tabela 4), não foram observadas associações estatisticamente significantes entre o consumo excessivo de álcool e a insatisfação com a imagem corporal, para o total da amostra ou na análise estratificada por sexo.

Dentre as variáveis de ajuste, na análise bivariada, considerando toda a amostra estudada, os seguintes fatores estavam positivamente associados com o consumo excessivo: não ter religião $(\mathrm{OR}=2,08$; IC95\%: 1,63-2,66), ter escolaridade menor ou igual a oito anos (OR = 1,53; IC95\%: 1,19$1,97)$ e composição familiar mononuclear/mora só ou com outras pessoas (OR = 1,97; IC95\%: 1,542,54), enquanto idade entre 15-19 anos (OR = 0,50; IC95\%: 0,39-0,64), ser solteiro/divorciado/viúvo 


\section{Tabela 4}

Modelo ajustado do consumo excessivo de álcool da população de adolescentes e jovens estratificado por sexo.

Camaçari, Bahia, Brasil, 2011-2012.

\begin{tabular}{|c|c|c|c|c|c|c|}
\hline \multirow[t]{2}{*}{ Características } & \multicolumn{2}{|r|}{ Total } & \multicolumn{2}{|c|}{ Masculino } & \multicolumn{2}{|c|}{ Feminino } \\
\hline & OR & IC95\% & OR & IC95\% & OR & IC95\% \\
\hline \multicolumn{7}{|l|}{ Insatisfação com a imagem } \\
\hline Não & 1,00 & - & 1,00 & - & 1,00 & - \\
\hline Sim & 0,86 & $0,64-1,18$ & 0,81 & $0,54-1,23$ & 1,27 & $0,76-2,13$ \\
\hline \multicolumn{7}{|l|}{ Idade (anos) } \\
\hline $20-24$ & 1,00 & - & 1,00 & - & 1,00 & - \\
\hline $15-19$ & 0,55 & $0,41-0,73$ * & 0,41 & $0,27-0,61$ * & 0,81 & $0,53-1,24$ \\
\hline \multicolumn{7}{|l|}{ Raça } \\
\hline Branca & 1,00 & - & 1,00 & - & 1,00 & - \\
\hline Negra/Parda/Índia/Amarela & 1,12 & $0,73-1,73$ & 0,87 & $0,47-1,61$ & 1,27 & $0,64-2,50$ \\
\hline \multicolumn{7}{|l|}{ Religião } \\
\hline Sim & 1,00 & 0,00 & 1,00 & 0,00 & 1,00 & 0,00 \\
\hline Não & 2,02 & $1,57-2,60$ * & 1,51 & $1,04-2,19$ * & 2,48 & $1,72-3,58$ * \\
\hline \multicolumn{7}{|l|}{ Estado Conjugal } \\
\hline Casado/Mora junto & 1,00 & - & 1,00 & - & 1,00 & - \\
\hline Solteiro/Divorciado/Viúvo & 0,91 & $0,64-1,30$ & 0,58 & $0,30-1,12$ & 0,92 & $0,58-1,47$ \\
\hline \multicolumn{7}{|l|}{ Trabalho } \\
\hline Sim & 1,00 & - & 1,00 & - & 1,00 & - \\
\hline Não & 0,61 & $0,47-0,80$ * & 0,55 & $0,38-0,81$ * & 1,09 & $0,71-1,70$ \\
\hline \multicolumn{7}{|l|}{ Escolaridade (anos) } \\
\hline$>8$ & 1,00 & - & 1,00 & - & 1,00 & - \\
\hline$\leq 8$ & 1,63 & $1,24-2,15$ * & 1,29 & $0,86-1,95$ & 1,77 & $1,19-2,64^{*}$ \\
\hline Filhos & & & & & & - \\
\hline Sim & 1 & - & 1 & - & 1 & - \\
\hline Não & 0,95 & $0,66-1,37$ & 0,82 & $0,40-1,66$ & 0,72 & $0,45-1,16$ \\
\hline \multicolumn{7}{|l|}{ Composição familiar } \\
\hline Nuclear & 1,00 & - & 1,00 & - & 1,00 & - \\
\hline Mononuclear/Mora só ou com outras pessoas & 1,61 & $1,22-2,13^{*}$ & 1,80 & $1,23-2,64^{*}$ & 1,50 & $0,98-2,30$ \\
\hline \multicolumn{7}{|l|}{ Número de amigos } \\
\hline Nenhum & 1,00 & - & 1,00 & - & 1,00 & - \\
\hline Pelo menos 1 amigo & 0,93 & $0,53-1,63$ & 0,77 & $0,27-2,17$ & 0,99 & $0,50-2,00$ \\
\hline
\end{tabular}

IC95\%: intervalo de 95\% de confiança; OR: odds ratio.

Fonte: inquérito domiciliar, Camaçari, 2011-2012.

* Resultado estatisticamente significativo.

No que concerne ao sexo feminino, os fatores não ter religião ( $O R=2,81$; IC95\%: 1,97-4,00), ter escolaridade menor ou igual a oito anos (OR = 2,15; IC95\%: 1,49-3,10) e composição familiar mononuclear/mora só ou com outras pessoas (OR = 2,03; IC95\%: 1,39-2,97) associaram-se positivamente ao consumo excessivo, enquanto que ser solteiro/divorciado/viúvo (OR = 0,58; IC95\%: 0,41-0,83) e não ter filhos $(\mathrm{OR}=0,49$; IC95\%: 0,34-0,70) associaram negativamente ao consumo excessivo de álcool (Tabela 3).

Após o ajuste do modelo, as variáveis que permaneceram estatisticamente significantes com consumo excessivo de álcool foram religião (OR = 2,02; IC95\%: 1,57-2,60), escolaridade (OR = 1,63; IC95\%: 1,24-2,15), composição familiar (OR = 1,61; IC95\%: 1,22-2,13), idade (OR = 0,55; IC95\%: 0,410,73) e trabalho $(\mathrm{OR}=0,61$; IC95\%: 0,47-0,80) (Tabela 4). 
Quando estratificadas por sexo masculino, as variáveis que permaneceram associadas ao consumo excessivo na população estudada foram idade (OR = 0,41; IC95\%: 0,27-0,61), religião $(\mathrm{OR}=1,51$; IC95\%: 1,04-2,19), trabalho (OR = 0,55; IC95\%: 0,38-0,81) e composição familiar (OR = 1,80; IC95\%: 1,23-2,64). Em relação ao sexo feminino, as variáveis que se mantiveram associadas positivamente ao consumo excessivo de álcool foram religião (OR = 2,48; IC95\%: 1,72-3,58) e escolaridade $(\mathrm{OR}=1,77$; IC95\%: 1,19-2,64) (Tabela 4).

\section{Discussão}

Os achados deste estudo não confirmaram a hipótese inicial da existência de associação entre o consumo excessivo e a insatisfação com a imagem corporal. Mesmo após o ajuste do modelo final, não foram encontradas associações estatisticamente significantes entre o consumo excessivo de álcool e a insatisfação com a imagem corporal tanto para o total dos indivíduos quanto na análise estratificada por sexo.

Os resultados foram semelhantes com um estudo nacional de desenho transversal realizado em 2008 com 236 estudantes universitários que frequentavam o curso de Educação Física em Santa Catarina, que, também, não encontrou associação entre a insatisfação com a imagem corporal e o consumo excessivo de álcool 40 . Outros estudos, entretanto, têm evidenciado essa associação no sexo feminino. Um estudo transversal, realizado com 6.863 adolescentes chineses, evidenciou que meninas insatisfeitas com a sua imagem corporal eram mais propensas ao consumo excessivo de álcool, entretanto, não foi observada associação estatisticamente significante para o sexo masculino ${ }^{41}$. Resultados de um estudo longitudinal desenvolvido com 937 escolares americanos revelou que meninas com menor satisfação corporal tinham 4,78 vezes mais chances de consumir álcool num padrão excessivo 42 .

Embora haja uma relação estabelecida entre imagem corporal, sobretudo para o controle de peso e exposição a comportamentos de risco, são poucos os estudos que tentam analisar uma possível relação entre consumo excessivo de álcool e a insatisfação com a imagem corporal 43. Pesquisas anteriores identificaram diferenças entre os sexos quanto à insatisfação corporal e estratégias para o controle de peso. Estudo desenvolvido com 4.746 escolares americanos observou que adolescentes do sexo feminino que relataram seguir uma dieta apresentaram maior chance para uso de substâncias psicoativas, entre elas, o consumo de álcool 44

Ainda que as análises deste estudo não tenham evidenciado associação entre os dois fenômenos estudados, os resultados deste estudo demonstraram altas prevalências de consumo excessivo de álcool e insatisfação com a imagem corporal entre adolescentes e jovens. A maioria dos entrevistados declarou estar insatisfeito com a imagem corporal, resultado que corrobora com outros achados científicos 10,40. As meninas estavam mais insatisfeitas com a imagem corporal do que os meninos, embora a diferença observada tenha sido pequena. Importante destacar os riscos para saúde associados a esse fenômeno, uma vez que, com a intenção de adequar a sua imagem corporal aos padrões de beleza vigente, os indivíduos podem desenvolver hábitos não saudáveis e transtornos alimentares ${ }^{45}$. Adicionalmente, as pequenas diferenças entre os sexos já vêm sendo evidenciadas na literatura científica, pois o sexo masculino, também, tem apresentado insatisfação com a sua imagem, mas, ao contrário das mulheres, geralmente, desejam aumentar as silhuetas e ser mais musculosos 10,45.

Analisando as covariáveis que se mostraram associadas com o consumo excessivo, destaca-se a maior prevalência de consumo no sexo masculino, uma associação bem estabelecida na literatura científica 26,27,46,47. Embora este estudo tenha evidenciado maior prevalência de consumo excessivo de álcool entre os jovens, a literatura aponta que esse hábito tem início cada vez mais precoce, como revelam dois estudos nacionais de corte transversal realizados em municípios do interior. O primeiro, em Minas Gerais, com 638 adolescentes, demonstrou que o consumo excessivo associou-se com idade do primeiro consumo (menor ou igual a 12 anos) e com a faixa etária entre 16-17 anos 17. O segundo, em São Paulo, com 515 indivíduos com idade igual ou superior aos 14 anos apontou que os jovens (entre 14-29 anos) apresentaram maiores prevalências de consumo excessivo quando comparados com os adultos e idosos 48 . Neste presente estudo, foi observado que a população entre 15-19 anos tem menor chance de consumir álcool num padrão excessivo quando comparada ao grupo de jovens entre 20-24 anos, possivelmente, devido à maior liberdade para aquisição da substância entre os jovens, visto que, no Brasil, a lei proíbe vendas e entregas de bebidas alcoólicas a crianças ou adolescentes, conforme disposto na Lei no 13.106, de 17 de março de 201549. 
Nesta pesquisa, foi observado que adolescentes e jovens que não trabalhavam tiveram menor chance de consumir álcool em excesso do que aqueles que trabalhavam, provavelmente porque quem trabalha tem renda própria e condições financeiras para aquisição da substância ${ }^{47}$. A exemplo disso, um estudo transversal desenvolvido com 48.115 adolescentes escolares, nas capitais brasileiras, revelou que estudantes com vínculos empregatícios tiveram 1,84 vez mais chance de consumir álcool num padrão excessivo quando comparados aos que não possuíam vínculo trabalhista 50 .

Os achados na literatura quanto à associação entre consumo de álcool e escolaridade são contraditórios 48. Nesta pesquisa, adolescentes e jovens com escolaridade menor ou igual a oito anos tiveram 1,63 mais chance de consumir álcool com padrão excessivo. Ao contrário, um estudo de base populacional realizado no interior de São Paulo com 515 adolescentes revelou que o maior número de abstinentes foi encontrado na população de menor escolaridade 48 .

No que concerne às características que envolvem contextos sociais e relação interpessoal, o consumo excessivo de álcool associou-se com composição familiar. Os resultados desta investigação sugerem que indivíduos que declararam composição familiar mononuclear, morar só ou com outras pessoas têm mais chances de consumir excessivamente o álcool. Esse achado é importante pelo fato de o consumo de álcool ser um comportamento moldado pelo ambiente social e familiar 51 e a influência quanto ao vínculo de moradia, que se refere à composição familiar atual, no desenvolvimento de comportamentos de risco e proteção à saúde 52 .

Finalmente, corroborando com a literatura, que tem destacado o engajamento religioso como fator protetor contra comportamento de risco 53 , no presente estudo, não possuir religião aumentou a chance de consumir álcool excessivamente entre a população de jovens e adolescentes. Um estudo longitudinal desenvolvido com 436 adolescentes em Belo Horizonte apontou que, quanto menor a frequência e o engajamento religioso, maiores as chances na frequência de consumo excessivo de álcool. Isso porque o ambiente religioso promove orientações morais e comportamentais que influenciam diretamente na redução dos comportamentos de risco 54 .

Para a cuidadosa interpretação dos resultados, algumas limitações do estudo precisam ser discutidas. Primeiro, as características inerentes ao desenho do estudo transversal, incluindo a causalidade reversa, limitam a possibilidade de inferência causal entre as variáveis. Segundo, o uso de métodos para mensuração autorreferidos, por meio de questionário, que são passíveis de viés de informação, pois o entrevistado pode omitir e/ou distorcer as suas respostas, o que pode resultar em subestimação ou superestimação dos resultados. Terceiro, no processo de amostragem, não foram utilizados pesos amostrais para calcular as probabilidades de seleção da microárea e domicílio, sendo adotada uma estratégia alternativa com análises que corrigem as estimativas usando o coeficiente de correlação intraclasse devido ao desenho do estudo. Por outro lado, como potencialidade do estudo, que o distingue da maioria das investigações nacionais com população de jovens e adolescentes, temos sua natureza de inquérito domiciliar com a possibilidade de levantamento dos fatores que estão associados ao consumo excessivo de álcool de uma população específica.

Embora o presente estudo não tenha evidenciado uma associação entre os fenômenos estudados, os resultados demonstraram altas prevalências tanto de consumo excessivo de álcool como de insatisfação com a imagem corporal entre adolescentes e jovens. Para a abordagem desses problemas, são necessárias políticas públicas com intervenções voltadas para esse grupo etário, tanto para a redução do consumo excessivo de álcool, que pode se associar a diversos problemas de saúde, inclusive o desenvolvimento de dependência, como ao que concerne à insatisfação corporal, que pode desencadear a aquisição de comportamentos de risco e de distúrbios alimentares, como é o caso de bulimia e anorexia, como também afetar a autoestima, podendo progredir para casos de depressão.

Esses cuidados podem ocorrer ainda na comunidade, como nos espaços da atenção básica à saúde e escolas do território, no âmbito de políticas a exemplo do Programa Saúde na Escola (PSE), instituído pelo Ministério da Saúde, desde o ano de 2007, uma ação política intersetorial, dos setores públicos Saúde e Educação, que visam promover saúde e educação integral aos adolescentes, jovens e crianças. Dessa forma, os resultados deste estudo chamam a atenção para a necessidade de articulação entre a Rede Municipal de Saúde e Educação em questão, de modo que os temas como imagem corporal e consumo de álcool sejam incluídos nas agendas políticas como meio de buscar estratégias para o enfrentamento de tais problemas. 


\section{Colaboradores}

B. E. B. Guimarães, R. Aquino, N. M. B. L. Prado e P. V. A. Rodrigues contribuíram substancialmente para concepção, planejamento, análise e interpretação dos dados do artigo; revisou criticamente o conteúdo intelectual importante; participou na aprovação final da versão a ser publicada e está de acordo para prestar contas de todos os aspectos do trabalho.

\section{Informações adicionais}

ORCID: Bárbara Emanuely de Brito Guimarães (0000-0001-7426-3311); Rosana Aquino (00000003-3906-5170); Nília Maria de Brito Lima Prado (0000-0001-8243-5662); Poliana Vieira Amaral Rodrigues (0000-0003-2513-7874).

\section{Agradecimentos}

Agradecimentos ao Conselho Nacional de Desenvolvimento Científico e Tecnológico (CNPq) por concessão da bolsa de estudo para alunos da pósgraduação (mestrado e doutorado).

\section{Referências}

1. Dumith SC, Menezes AMB, Bielemann RM, Petresco S, Silva ICM, Linhares RS, et al. Insatisfação corporal em adolescentes: um estudo de base populacional. Ciênc Saúde Colet 2012; 17:2499-505.

2. Fingeret MC, Gleaves DH, Pearson CA. On the methodology of body image assessment: the use of figural rating scales to evaluate body dissatisfaction and the ideal body standards of women. Body Image 2004; 1:207-12.

3. Petroski EL, Pelegrini A, Glaner MF. Motivos e prevalência de insatisfação com a imagem corporal em adolescentes. Ciênc Saúde Colet 2012; 17:1071-77.

4. Alvarenga MS, Philippi ST, Lourenço BH, Sato PM, Scagliusi FB. Insatisfação com a imagem corporal em universitárias brasileiras. J Bras Psiquiatr 2010; 59:44-51.

5. Papalia D, Feldman RD. Desenvolvimento físico e cognitivo na adolescência. In: Papalia D, Feldman RD, organizadores. Desenvolvimento humano. 12a Ed. Porto Alegre: Editora Artmed; 2013. p. 384-419.

6. Iepsen AM, Silva MC. Prevalência e fatores associados à insatisfação com a imagem corporal de adolescentes de escolas do Ensino Médio da zona rural da região sul do Rio Grande do Sul, 2012. Epidemiol Serv Saúde 2014; 23:317-25.

7. Mikolajczak J, Piotrowska E, Biernat J, Wyka J, Żechałko-Czajkowska A. Self-perceived assessment of nutritional status as a determinant of health-promoting and anti-health-promoting behaviors of adolescent boys. Adv Clin Exp Med 2012; 21:225-33.

8. Martins DF, Nunes MFO, Noronha APP. Satisfação com a imagem corporal e autoconceito em adolescentes. Psicol Teor Prát 2008; 10:94-105.

9. Silva SU, Barufaldi LA, Andrade SSCA, Santos MAS, Claro RM. Estado nutricional, imagem corporal e associação com comportamentos extremos para controle de peso em adolescentes brasileiros, Pesquisa Nacional de Saúde do Escolar de 2015. Rev Bras Epidemiol 2018; 21 Suppl 1:e180011.

10. Rodrigues PVA. Relações entre imagem corporal e autoestima em uma população de adolescentes e jovens em um município da Bahia [Dissertação de Mestrado]. Salvador: Instituto de Saúde Coletiva, Universidade Federal da Bahia; 2015.

11. Malta DC, Mascarenhas MDM, Porto DL, Barreto SM, Morais Neto OL. Exposição ao álcool entre escolares e fatores associados. Rev Saúde Pública 2014; 48:52-62.

12. Martini MCS, Assumpção D, Barros MBA, Canesqui AM, Barros Filho AA. Are normalweight adolescents satisfied with their weight? São Paulo Med J 2016; 134:219-27.

13. Andrew R, Tiggemann M, Clark L. Positive body image and young women's health: implications for sun protection, cancer screening, weight loss and alcohol consumption behaviours. J Health Psychol 2016; 21:28-39. 
14. Nelson MC, Lust k, Story M, Ehlinger E. Alcohol use, eating patterns, and weight behaviors in a university population. Am J Health Behav 2009; 33 Suppl 1:S227-37.

15. Littleton H, Breitkopf CR, Berenson A. Body image and risky sexual behaviors: an investigation in a tri-ethnic sample. Body Image 2005; 2:193-8.

16. World Health Organization. Global status report on alcohol and health. https://www.who. int/substance_abuse/publications/global_al cohol_report/en/ (acessado em 19/Dez/2018).

17. Reis TG, Oliveira LCM. Padrão de consumo de álcool e fatores associados entre adolescentes estudantes de escolas públicas em município do interior brasileiro. Rev Bras Epidemiol 2015; 18:13-24.

18. Siliquini R, Colombo A, Berchialla P, Bert F. Binge drinking and psychoactive drug use in a cohort of european youths. J Public Health Res 2012; 1:83-8.

19. Brown SA, McGue M, Maggs J, Schulenberg J, Hingson R, Swartzwelder S, et al. Underage alcohol use summary of developmental processes and mechanisms: ages 16-20. Alcohol Res Health 2009; 32:41-52.

20. Munhoz TN, Santos IS, Nunes BP, Mola CL, Silva ICM, Matijasevich A. Tendências de consumo abusivo de álcool nas capitais brasileiras entre os anos de 2006 a 2013: análise das informações do VIGITEL. Cad Saúde Pública 2017; 33:e00104516.

21. Bertolote JM. Glossário de álcool e drogas. Brasília: Secretaria Nacional de Políticas sobre Drogas; 2010.

22. Martinotti G, Lupi M, Carlucci L, Santacroce $\mathrm{R}$, Cinosi E, Acciavatti, T, et al. Alcohol drinking patterns in young people: a survey-based study. J Health Psychol 2017; 22:1889-96.

23. Veiga LDB, Santos VC, Santos MG, Ribeiro JF, Amaral ASN, Nery AA, et al. Prevalência e fatores associados à experimentação e ao consumo de bebidas alcoólicas entre adolescentes escolares. Cad Saúde Colet (Rio J.) 2016; 24: 368-75.

24. Garcia LP, Freitas LRS. Consumo abusivo de álcool no Brasil: resultados da Pesquisa $\mathrm{Na}$ cional de Saúde 2013. Epidemiol Serv Saúde 2015; 24:227-37.

25. Ferreira LN, Bispo Júnior JP, Sales ZN, Casotti CA, Braga Junior ACR. Prevalência e fatores associados ao consumo abusivo e à dependência de álcool. Ciênc Saúde Colet 2013; 18:3409-18.

26. Chartier KG, Hesselbrock MN, Hesselbrock VM. Alcohol problems in young adults transitioning from adolescence to adulthood: the association with race and gender. Addict Behav 2011; 36:167-74.

27. Malta DC, Mascarenhas MDM, Porto DL, Duarte EA, Sardinha LM, Barreto SM, et al. Prevalência do consumo de álcool e drogas entre adolescentes: análise dos dados da Pesquisa Nacional de Saúde Escolar. Rev Bras Epidemiol 2011; 14 Suppl 1:S136-46.
28. Grucza RA, Norberg KE, Bierut LJ. Binge drinking among youths and young adults in the United States: 1979-2006. J Am Acad Child Adolesc Psychiatr 2009; 48:692-702.

29. Toumbourou JW, Evans-Whipp TJ, Smith R, et al. Adolescent predictors and environmental correlates of young adult alcohol use problems. Addiction 2014; 109:417-24.

30. Eichen DM, Conner BT, Daly BP, Fauber RL. Weight perception, substance use, and disordered eating behaviors: comparing normal weight and overweight high-school students. J Youth Adolesc 2012; 41:1-13.

31. Aquino R. Avaliação dos efeitos da Estratégia Saúde da Família: adoção de hábitos saudáveis e acessibilidade a serviços primários de promoção da saúde e prevenção de riscos e agravos. Relatório final do projeto. Salvador: Instituto de Saúde Coletiva, Universidade Federal da Bahia; 2012.

32. Méndez EB. Uma versão brasileira do AUDIT (Alcohol Use Disorders Identification Test) [Dissertação de Mestrado]. Pelotas: Departamento de Medicina Social, Universidade Federal de Pelotas; 1999.

33. Lima CT, Freire AC, Silva AP, Teixeira RM, Farrell M, Prince M. Concurrent and construct validity of the audit in an urban brazilian sample. Alcohol 2005; 40:584-89.

34. Babor TF, Higgins-Biddle JC, Saunders JB, Monteiro MG. AUDIT: the Alcohol Use Disorders Identification Test: guidelines for use in primary health care. Geneva: World Health Organization; 2001.

35. Kakeshita IS, Silva AIP, Zanatta DP, Almeida SS. Construção e fidedignidade teste-reteste de escalas de silhuetas brasileiras para adultos e crianças. Psicol Teor Pesqui 2009; 25:263-70.

36. Laus MF, Almeida SS, Murarole MB, BragaCosta TM. Estudo de validação e fidedignidade de escalas de silhuetas brasileiras em adolescentes. Psicol Teor Pesqui 2013; 29:403-09.

37. Campana ANNB, Tavares MCGCF. Avaliação da imagem corporal: instrumentos e diretrizes para a pesquisa. São Paulo: Phorte; 2009.

38. Rao JNK, Scott AJ. The analysis of categorical data from complex sample surveys: chisquared tests for goodness of fit and independence in two-way tables. J Am Stat Assoc 1981; 76:221-30.

39. Rao JNK, Scott AJ. On chi-squared tests for multiway contingency tables with cell proportions estimated from survey data. Ann Stat 1984; 12:46-60.

40. Ferrari EP, Petroski EL, Silva DAS. Prevalence of body image dissatisfaction and associated factors among physical education students. Trends Psychiatry Psychother 2013; 35:11927.

41. Xie B, Chou CP, Spruijt-Metz D, Reynolds K, Clark F, Palmer PH, et al. Weight perception and weight-related sociocultural and behavioral factors in Chinese adolescents. Prev Med 2006; 42:229-34. 
42. Jones A, Winter VR, Pekarek, E, Walters J. Binge drinking and cigarette smoking among teens: does body image play a role? Children Youth Serv Rev 2018; 91:232-36.

43. Ramseyer Winter V, Kennedy AK, O’Neill E. Adolescent tobacco and alcohol use: the influence of body image. Journal of Child \& Adolescent Substance Abuse 2017; 26:219-28.

44. Crow S, Eisenberg ME, Story M, NeumarkSztainer D. Psychosocial and behavioral correlates of dieting among overweight and nonoverweight adolescents. J Adolesc Health 2006; 38:569-74.

45. Claro RM, Santos MAS, Oliveira MC. Imagem corporal e atitudes extremas em relação ao peso em escolares brasileiros (PeNSE 2012). Rev Bras Epidemiol 2014; Suppl 1:S146-57.

46. Dallo L, Martins RA. Associação entre as condutas de risco do uso de álcool e sexo desprotegido em adolescentes numa cidade do Sul do Brasil. Ciênc Saúde Colet 2018; 23:303-14.

47. Campos JADB, Almeida JC, Garcia PPNS, Faria JB. Consumo de álcool entre estudantes do ensino médio do Município de Passos - MG. Ciênc Saúde Colet 2011; 16: 4745-54.

48. Barros MBA, Marín-León L, Oliveira HB, Dalgalarrondo P, Botega NJ. Perfil do consumo de bebidas alcoólicas: diferenças sociais e demográficas no Município de Campinas, Estado de São Paulo, Brasil, 2003. Epidemiol Serv Saúde 2008; 17:259-70.

49. Brasil. Lei no 13.106, de 17 de março de 2015. Altera a Lei no 8.069, de 13 de julho de 1990 - Estatuto da Criança e do Adolescente, para tornar crime vender, fornecer, servir, ministrar ou entregar bebida alcoólica a criança ou a adolescente; e revoga o inciso I do art. 63 do Decreto-Lei no 3.688, de 3 de outubro de 1941 - Lei das Contravenções Penais. Lei Estatuto da Criança e do Adolescente de 2015, Pub L N.o 13.106 (17 de março de 2015). Diário Oficial da União 2015; 18 mar.
50. Galduróz JCF, Sanchez ZM, Opaleye ES, Noto AR, Fonseca AM, Gomes PLS, et al. Fatores associados ao uso pesado de álcool entre estudantes das capitais brasileiras. Rev Saúde Pública 2010; 44:267-73.

51. Surkan P, Fielding-Miller R, Melchior M. Parental relationship satisfaction in French young adults associated with alcohol abuse and dependence. Addict Behav 2012; 37:31317.

52. Matos AM, Carvalho RC, Costa MCOl, Gomes KEPS, Santos LM. Consumo frequente de bebidas alcoólicas por adolescentes escolares: estudo de fatores associados. Rev Bras Epidemiol 2010; 13:302-13.

53. Mellor JM, Freeborn BA. Religious participation and risky health behaviors among adolescents. Health Econ 2011; 20:1226-40.

54. Jorge KO, Ferreira RC, Ferreira EF, Vale MP, Kawachi I, et al. Binge drinking and associated factors among adolescents in a city in southeastern Brazil: a longitudinal study. Cad Saúde Pública 2017; 33:e00183115. 


\section{Abstract}

The study aimed to analyze the association between excessive alcohol intake and dissatisfaction with one's body image in a population of adolescents and young adults. This was a cross-sectional study with a household survey design in a sample of 1,582 individuals 15 to 24 years of age living in Camaçari, Bahia State, Brazil. Excessive alcohol intake was identified by the Alcohol Use Disorder Identification Test (AUDIT), and dissatisfaction with body image was measure with the Silhouette Figure Scale. Confounding variables were: sex, age, race, schooling, work, religion, family composition, number of close friends, marital status, and children. The association between excessive alcohol intake and body dissatisfaction was estimated by logistic regression. Prevalence of excessive alcohol intake was 21.9\% (higher in males), and prevalence of body image dissatisfaction was $79.5 \%$ (higher in females). No statistically significant association was found between excessive alcohol intake and dissatisfaction with body image. After adjustment of the final model, the variables that remained associated with excessive alcohol intake were religion $(O R=2.02)$, schooling $(O R=1.63)$, family composition $(O R=1.61)$, age $(O R=0.55)$, and work $(O R=0.61)$. Although the analyses did not show an association between excessive alcohol intake and dissatisfaction with body image, the study's results call attention to the high prevalence rates for the two phenomena. This shows the need to develop interventions focused on practices of care for this specific population.

Underage Drinking; Adolescent; Young Adult; Body Image

\section{Resumen}

El estudio tiene como objetivo analizar la asociación entre el consumo excesivo de alcohol y la insatisfacción con la imagen corporal en una población de adolescentes y jóvenes. Se trata de un estudio transversal, de tipo encuesta domiciliaria, realizada a 1.582 individuos en la franja etaria de 15 a 24 años, residentes en Camaçari, Bahía, Brasil. El consumo excesivo de alcohol fue identificado por el Alcohol Use Disorder Identification Test (AUDIT) y la insatisfacción con la imagen corporal mediante la Escala de Figura de Siluetas. Las variables confusoras fueron: sexo, edad, raza, escolaridad, trabajo, religión, estructura familiar, números de amigos cercanos, estado conyugal e hijos. La asociación entre el consumo excesivo y la insatisfacción corporal se estimó mediante regresión logística. La prevalencia del consumo excesivo de alcohol fue de un 21,9\%, siendo mayor entre el sexo masculino, $y$ de insatisfacción con la imagen corporal fue de un 79,5\%, siendo mayor entre el sexo femenino. No se encontró una asociación estadísticamente significativa entre consumo excesivo de alcohol e insatisfacción con la imagen corporal. Tras el ajuste del modelo final, las variables que permanecieron asociadas con el consumo excesivo fueron: religión $(O R=2,02)$, escolaridad $(O R=1,63)$, estructura familiar $(O R=1,61)$, edad $(O R=0,55)$ y trabajo $(O R=0,61)$. A pesar de que los análisis no evidenciaron la asociación entre el consumo excesivo de alcohol e insatisfacción con la imagen corporal, los resultados de este estudio llaman la atención por las altas prevalencias respecto a los dos fenómenos. De esta forma, se evidencia la necesidad del desarrollo de intervenciones dirigidas a prácticas de cuidado para esta población especifica.

Consumo de Alcohol en Menores; Adolescente; Adulto Joven; Imagen Corporal
Recebido em 06/Mar/2019

Versão final reapresentada em 14/Jun/2019

Aprovado em 04/Jul/2019 